\title{
Editorial
}
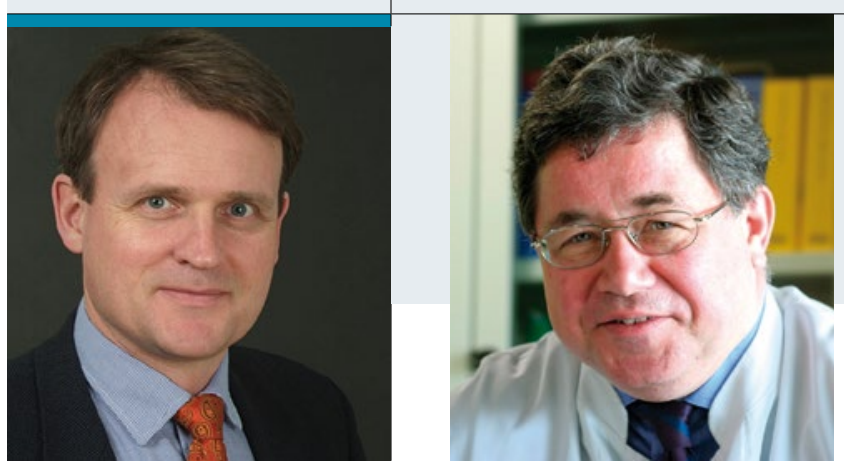

"Um weiter Klarheit in die Thematik zu

bringen, haben sich AeDA und DGAKI an das

Paul-Ehrlich-Institut gewendet, um sich nach

dem aktuellen Stand der TAV zu erkundigen."

\section{Licht ins Dunkel der TAV}

n jüngster Vergangenheit ist es durch die Aussagen einiger Prüfgremien, KVen und Krankenkassen in der Ärzteschaft zu einer Verunsicherung bezüglich der Verordnungsfähigkeit von gewissen Präparaten zur spezifischen Immuntherapie gekommen. Bestimmte Präparate sollen angeblich trotz ihrer Verkehrsfähigkeit und Verfügbarkeit durch fehlende Evidenz ihrer Wirksamkeit eine erhöhte Gefahr von Arzneimittelregressen für den verordneten Arzt heraufbeschwören. Dass es sich bei dieser Beurteilung um eine eindeutig korrekturbedürftige Fehleinschätzung handelt, hatten wir bereits in einer Stellungnahme der allergologischen Fachgesellschaften AeDA, DGAKI und GPA im Dezemberheft des Allergo Journal herausgestellt und im Anschluss in einem Positionspapier der Gesellschaften noch einmal auf den Unterschied zwischen Evidenz und Wirksamkeit hingewiesen [Brehler et al. Allergo J Int 2016;25:38-43]. Um weiter Klarheit in diese Thematik zu bringen, haben sich nun die allergologischen Fachgesellschaften in einem offenen Brief an das Paul-Ehrlich-Institut (PEI) gewendet, um sich nach dem aktuellen Stand der Umsetzung der Therapieallergene-Verordnung (TAV) zu erkundigen. In dieser Ausgabe können Sie die Anfragen und die Antworten des PEI nachlesen, die im Wesentlichen die Positionen der Fachgesellschaften bestätigen. In Kürze wird hier festgestellt, dass es innerhalb des Übergangsprozesses der TAV nicht möglich ist, vernünftig die Evidenzlevel einzelner Produkte zu berücksichtigen und ein Produkt erst nach Abschluss des gesamten Prüfprogramms beurteilt werden kann. Zusätzlich wurde bestätigt, dass aktuell keine Produkte auf dem Markt sind, für die kein Studienprogramm vorgelegt wurde, und dass diejenigen Präparate, für die derzeit noch laufende Anträge auf Zulassung unter den Übergangsvorschriften der TAV vorliegen, rechtskonform in den Verkehr gebracht werden. Eine Einschätzung der Erstattungsfähigkeit liegt nicht im Aufgabenbereich des PEI. Sollten Präparate, die aktuell noch in der Übergangsphase verkehrsfähig sind, Gründe aufzeigen, die behördliche Grundlage der Verkehrs- fähigkeit zu entziehen, so wird dies auch erfolgen. Weitere Details hierzu finden Sie auf den Seiten 60 bis 63 der aktuellen Ausgabe.

Ebenfalls auf den Verbandsseiten finden Sie wieder das Update zum Flickenteppich der allergologischen Vergütungen. Erfreulicherweise werden in zunehmend mehr KV-Bezirken allergologische Leistungen als förderungswürdig eingestuft (S. 58).

In unserer letzten Ausgabe hatten wir die Bedeutung des Zucker-Epitops Alpha-Gal bei der Fleischallergie vom verzögerten Typ aus amerikanischer, schwedischer und deutscher Sicht dargestellt. In der aktuellen Ausgabe finden Sie zwei spannende Übersichtsarbeiten, die über Fleischallergien unabhängig von der Alpha-Gal-Sensibilisierung berichten (S. 26 / S. 30). Lassen Sie sich in die Welt des VogelEi-Syndroms und des Schwein-Katzen-Syndroms entführen und genießen Sie die ausgezeichnet aufgearbeitete Kasuistik einer Allergie auf Pferdefleisch, die offensichtlich durch eine primäre Sensibilisierung gegen Hundeepithelien vermittelt wurde. Diese englische Erstbeschreibung des PferdHund-Syndroms finden Sie auch in einer deutschen Zusammenfassung unter der Rubrik „Literatur kompakt“. Ein absolutes Muss für unsere Leser ist die Lektüre der Kurzfassung der aktuellen Neurodermitis-Leitlinie, die auf wenigen Seiten die wesentlichen Aussagen der über 100-seitigen Leitlinie zusammenfasst (S. 36). Dieser Beitrag eignet sich besonders gut zur CME-Fortbildung und ist mit vier CME-Punkten zertifiziert.

$\mathrm{Zu}$ guter Letzt nochmals eine Bitte von uns an unsere Leser und Autoren: Denken Sie daran, Arbeiten aus unserem Verbandsjournal zu zitieren und falls Sie zitieren, bitte zitieren Sie die Literaturstelle aus dem Allergo Journal International, damit es sich für die Bewertung unseres Verbandsjournals positiv auswirkt. Viel Freude bei der aktuellen Ausgabe!

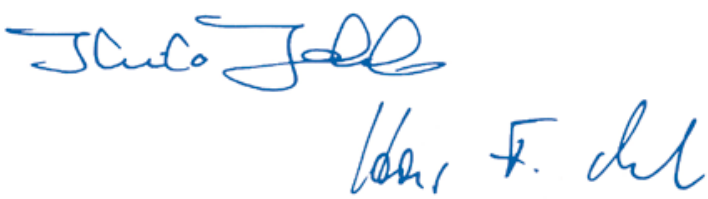

